\title{
Pseudodifferential Operators and Equations of Variable Order
}

\author{
V. B. Vasilyev \\ Belgorod National Research University, Belgorod, 308015 Russia \\ e-mail:vbv57@inbox.ru
}

Received April 6, 2018

\begin{abstract}
A new class of elliptic pseudodifferential equations of variable order is considered. Local Sobolev-Slobodetskii spaces are introduced and used to obtain theorems on the continuity of these pseudodifferential operators and describe the Fredholm properties of the corresponding pseudodifferential equations and boundary value problems.
\end{abstract}

DOI: $10.1134 /$ S0012266118090045

\section{INTRODUCTION}

So far, the theory of pseudodifferential operators, equations, and the corresponding boundary value problems has a very rich history [1-4]. In the recent years, the theory of pseudodifferential operator and equations on manifolds with singularities or with singularities on the boundary attracts constantly increasing attention [5-7]. To study the Fredholm property of elliptic pseudodifferential equations on compact manifolds with nonsmooth boundary, we proposed a special factorization of elliptic symbols, which was called the wave factorization [8] and which is based on the use of spaces of analytic functions in tube domains over cones [9].

Elliptic pseudodifferential equations in multidimensional domains $D \subset \mathbb{R}^{m}$ with nonsmooth boundary $\partial D$ were studied in $[8,10]$. The class of considered symbols was distinguished by the condition

$$
c_{1}(1+|\xi|)^{\alpha} \leq|A(x, \xi)| \leq c_{2}(1+|\xi|)^{\alpha},
$$

where $c_{1}$ and $c_{2}$ are positive constants. The number $\alpha \in \mathbb{R}$ was called the order of the pseudodifferential operator (symbol). In the present paper, we show that these methods can be applied to much more general classes of pseudodifferential equations.

The paper is organized as follows: we define local Sobolev-Slobodetskii spaces, introduce and study pseudodifferential operators of variable order, and describe the Fredholm property. In conclusion, we consider several boundary value problems.

\section{SPACES OF VARIABLE ORDER}

\subsection{Local Sobolev-Slobodetskii Spaces}

Let $s: \mathbb{R}^{m} \rightarrow \mathbb{R}$ be an arbitrary function satisfying the following two conditions.

1. There exists a finite limit $\lim _{|x| \rightarrow+\infty} s(x)$.

2. The function $s(x)$ satisfies the Lipschitz condition on $\mathbb{R}^{m}$; i.e., there exists a constant $C>0$ such that $\left|s\left(x_{1}\right)-s\left(x_{2}\right)\right| \leq c\left|x_{1}-x_{2}\right|$ for any $x_{1}, x_{2} \in \mathbb{R}^{m}$.

Definition 1. Assume that a function $s$ satisfying conditions (1) and (2) and a point $x \in \mathbb{R}^{m}$ are fixed. The local Sobolev-Slobodetskii space $H^{s(x)}\left(\mathbb{R}^{m}\right)$ consists of (generalized) functions $u$ for which the following expression is finite:

$$
\|u\|_{s(x)} \equiv\left(\int_{\mathbb{R}^{m}}(1+|\xi|)^{2 s(x)}|\tilde{u}(\xi)|^{2} d \xi\right)^{1 / 2},
$$

where $\tilde{u}$ is the Fourier transform of the function $u$. 
The function $\|u\|_{s(x)}$ is called the local $H^{s}$-norm of the function $u$.

For brevity, we write $H^{s(x)}$ instead of $H^{s(x)}\left(\mathbb{R}^{m}\right)$, and if we deal with $H^{s(x)}$-functions supported in a certain domain $D \subset \mathbb{R}^{m}$, then we write $H^{s(x)}(D)$.

Since in what follows we compare different operators in different local spaces, we need to choose a universal superspace where one can put all local Sobolev-Slobodetskii spaces, and a universal subspace contained in all local Sobolev-Slobodetskii spaces.

Definition 2. The universal Sobolev-Slobodetskii superspace $H^{s_{M}}$ is the space containing all local Sobolev-Slobodetskii spaces with finite norm $\|u\|_{s_{m}}=\inf _{x \in \mathbb{R}^{m}}\|u\|_{s(x)}$.

The universal Sobolev-Slobodetskii subspace $H^{s_{m}}$ is the space contained in all local SobolevSlobodetskii spaces with finite norm $\|u\|_{s_{M}}=\sup _{x \in \mathbb{R}^{m}}\|u\|_{s(x)}$.

Obviously,

$$
\|u\|_{s_{m}} \leq\|u\|_{s(x)} \leq\|u\|_{s_{M}} \quad \text { for any } \quad x \in \mathbb{R}^{m}
$$

\subsection{Properties}

By $S\left(\mathbb{R}^{m}\right)$ we denote the Schwartz class of infinitely differentiable functions rapidly decaying at infinity; this class forms a dense set in each local Sobolev-Slobodetskii space [4, p. 44]. Let $u \in S\left(\mathbb{R}^{m}\right)$. We compare two local norms $\|u\|_{s\left(x_{1}\right)}$ and $\|u\|_{s\left(x_{2}\right)}$.

Lemma 1. The following estimates hold:

$$
\begin{aligned}
\left|(1+|\xi|)^{s_{1}}-(1+|\xi|)^{s_{2}}\right| & \leq \ln (1+|\xi|)\left|s_{1}-s_{2}\right|(1+|\xi|)^{l} \quad \text { for some } \quad l \in\left[s_{1}, s_{2}\right] \\
\left|\|u\|_{s\left(x_{1}\right)}-\|u\|_{s\left(x_{2}\right)}\right| & \leq c_{1}\left|x_{1}-x_{2}\right|\|u\|_{l_{1}} \quad \text { for some } \quad l_{1} \in \mathbb{R}
\end{aligned}
$$

where $c_{1}$ is a constant.

Proof. The first inequality readily follows from the finite increment formula applied to a powerlaw function. The proof of the second inequality is simple as well. Indeed, by the definition of the $H^{s}$-norm,

$$
\|u\|_{s\left(x_{1}\right)}-\|u\|_{s\left(x_{2}\right)}=\left(\left.\int_{\mathbb{R}^{m}}\left|(1+|\xi|)^{2 s\left(x_{1}\right)}\right| \tilde{u}(\xi)\right|^{2} d \xi\right)^{1 / 2}-\left(\int_{\mathbb{R}^{m}}(1+|\xi|)^{2 s\left(x_{2}\right)}|\tilde{u}(\xi)|^{2} d \xi\right)^{1 / 2},
$$

and then, with regard to the properties of the power-law function and the first inequality, we obtain

$$
\begin{aligned}
\left\|\left|u\left\|_{s\left(x_{1}\right)}-\right\| u \|_{s\left(x_{2}\right)}\right|\right. & \leq\left(\int_{\mathbb{R}^{m}}\left|(1+|\xi|)^{2 s\left(x_{1}\right)}-(1+|\xi|)^{2 s\left(x_{2}\right)}\right||\tilde{u}(\xi)|^{2} d \xi\right)^{1 / 2} \\
& \leq\left|s\left(x_{1}\right)-s\left(x_{2}\right)\right|\left(\int_{\mathbb{R}^{m}} \ln (1+|\xi|)(1+|\xi|)^{2 l}|\tilde{u}(\xi)|^{2} d \xi\right)^{1 / 2} \leq c_{1}\left|x_{1}-x_{2}\right|\|u\|_{s_{M}},
\end{aligned}
$$

because the function $s(x)$ satisfies the Lipschitz condition. The proof of the lemma is complete.

Remark 1. Needless to say, such constructions of (Sobolev, Hölder, and $L_{p}$ ) spaces with variable exponent have already been used in previous studies. The Vishik-Eskin paper [11] dedicated to elliptic pseudodifferential equations was the first to do this, but the variable exponent has also appeared in a different context [12-16].

Consider a family of Hilbert spaces $H_{x}$ parametrized by points $x \in \mathbb{R}^{m}$. In particular, one can take $H_{x}=H^{s(x)}$. We denote the norm of an element $u \in H^{s(x)}$ by $\|u\|_{x}$ and assume that $S$ is a subset everywhere dense in $H^{s(x)}$ for any $x \in \mathbb{R}^{m}$. For $u \in S$, we define the functional

$$
f(x, u)=\|u\|_{x}
$$

DIFFERENTIAL EQUATIONS $\quad$ Vol. $54 \quad$ No. 92018 
Definition 3. A family of Hilbert spaces $\left\{H_{x}\right\}_{x \in \mathbb{R}^{m}}$ is said to be locally continuous at a point $x_{0} \in \mathbb{R}^{m}$ if for each fixed element $u \in S$ the functional $f(x, u)$ is continuous at the point $x_{0}$.

Lemma 2. The family of spaces $H^{s(x)}$ is locally continuous.

Proof. The assertion of the lemma follows from Lemma 1 and the fact that the class $S\left(\mathbb{R}^{m}\right)$ is dense in any space $H^{s(x)}$.

\section{OPERATORS OF VARIABLE ORDER}

A pseudodifferential operator in $\mathbb{R}^{m}$ is introduced $[1,3,17]$ as follows.

Definition 4. Let a function $A(x, \xi)$ locally integrable with respect to $\xi$ be given on $\mathbb{R}^{m} \times \mathbb{R}^{m}$. The pseudodifferential operator $A$ with symbol $A(x, \xi)$ is the operator of the form

$$
(A u)(x)=\iint_{\mathbb{R}^{m}} \int_{\mathbb{R}^{m}} A(x, \xi) e^{i(x-y) \xi} u(y) d y d \xi, \quad x \in \mathbb{R}^{m} .
$$

The function $A(x, \xi)$ is called the symbol of the operator $A$.

Let us give an equivalent definition of pseudodifferential operator in terms of its kernel, which was first used in [18]. By

$$
B(x, y)=F_{\xi \rightarrow y}^{-1} A(x, \xi)
$$

we denote the inverse Fourier transform (in the sense of generalized functions) of the symbol $A(x, \xi)$ with respect to the variable $\xi$. Then formula (1) can be written as

$$
(A u)(x)=\int_{\mathbb{R}^{m}} B(x, x-y) u(y) d y, \quad x \in \mathbb{R}^{m} .
$$

Formula (2) is called an integral representation of the operator $A$.

In the study of Fredholm properties of the operator $A$, a key role is played by the so-called local principle by which it is necessary to determine the invertibility conditions for "model" operators in "canonical" domains (for this, see [19, 20]). In this situation, the model operator is an operator whose symbol is independent of the spatial variable, and since we use a Hilbert scale of spaces, we give an equivalent definition of such an operator in Fourier transforms, which is convenient for our purposes as will be shown below.

Definition 5. A pseudodifferential operator $A$ with symbol $A(\cdot, \xi)$ is an operator of the form $(\widetilde{A u})(\xi)=A(\cdot, \xi) \tilde{u}(\xi)$.

\subsection{Class of Symbols}

Assume that a function $\alpha: \mathbb{R}^{m} \rightarrow \mathbb{R}$ has the same properties (1) and (2) as the function $s(x)$.

Definition 6. The symbol class $E_{\alpha(x)}$ consists of functions $A(x, \xi)$ defined on $\mathbb{R}^{m} \times \mathbb{R}^{m}$ and satisfying the following conditions: one has the double inequality

$$
c_{1}(1+|\xi|)^{\alpha(x)} \leq|A(x, \xi)| \leq c_{2}(1+|\xi|)^{\alpha(x)},
$$

where $c_{1}$ and $c_{2}$ are positive constants, and for any point $x_{0} \in \mathbb{R}^{m}$ there exists a neighborhood $U_{x_{0}}$ such that for all $x \in U_{x_{0}}$ the inequality

$$
\left|A(x, \xi)-A\left(x_{0}, \xi\right)\right| \leq c_{3}\left|x-x_{0}\right|(1+|\xi|)^{\alpha(x)}
$$

holds, where $c_{3}$ is a positive constant. The function $\alpha: \mathbb{R}^{m} \rightarrow \mathbb{R}$ is called the variable order of the pseudodifferential operator (symbol). 
Remark 2. It is assumed in inequality (4) that the point $x_{0}$ is located in a finite domain of the space $\mathbb{R}^{m}$. If $x_{0}$ is the point at infinity, then instead of inequality (4) it is necessary to take the inequality

$$
|A(x, \xi)-A(\infty, \xi)| \leq c_{4}|x|^{-1}(1+|\xi|)^{\alpha(x)} .
$$

Let us fix $x_{0} \in \mathbb{R}^{m}$. The operator with symbol $A\left(x_{0}, \xi\right)$ will be denoted by $A_{x_{0}}$. Then, with regard to Definition 3, one can readily obtain the following result.

Lemma 3. If $A\left(x_{0}, \xi\right) \in E_{\alpha\left(x_{0}\right)}$, then the operator $A_{x_{0}}$ is bounded in the local Sobolev-Slobodetskii space, $A_{x_{0}}: H^{s\left(x_{0}\right)} \rightarrow H^{s\left(x_{0}\right)-\alpha\left(x_{0}\right)}$.

Proof. Indeed, with regard to inequality (3), we have the obvious estimate

$$
\left\|A_{x_{0}} u\right\|_{s\left(x_{0}\right)-\alpha\left(x_{0}\right)}^{2}=\int_{\mathbb{R}^{m}}\left|A\left(x_{0}, \xi\right) \tilde{u}(\xi)\right|^{2}(1+|\xi|)^{2\left(s\left(x_{0}\right)-\alpha\left(x_{0}\right)\right)} d \xi \leq C \int_{\mathbb{R}^{m}}|\tilde{u}(\xi)|^{2}(1+|\xi|)^{2 s\left(x_{0}\right)} d \xi,
$$

which implies the boundedness of the operator in question. The proof of the lemma is complete.

This property of pseudodifferential operators is called the local boundedness.

Passing to the least upper bound in inequality (5), we see that each operator $A_{x_{0}}$ boundedly acts in the space $H^{s_{M}}, A_{x_{0}}: H^{s_{M}} \rightarrow H^{(s-\alpha)_{M}}$.

Definition 7. An operator $A$ with symbol $A(x, \xi)$ is said to be locally bounded if, for each point $x_{0} \in \mathbb{R}^{m}$, the operator $A_{x_{0}}: H^{s\left(x_{0}\right)} \rightarrow H^{s\left(x_{0}\right)-\alpha\left(x_{0}\right)}$ with symbol $A\left(x_{0}, \xi\right)$ is bounded.

Theorem 1. If $A(x, \xi) \in E_{\alpha(x)}$, then the function $x_{0} \mapsto\left\|A_{x_{0}}\right\|_{s\left(x_{0}\right)}$ is continuous at each point $x_{0} \in \mathbb{R}^{m}$, including the point at infinity.

Proof. Let us compare two operators $A_{x_{1}}$ and $A_{x_{2}}$ under the assumption that the points $x_{1}$ and $x_{2}$ are sufficiently close to each other. For $u \in S\left(\mathbb{R}^{m}\right)$, we have

$$
\left.\left.\widetilde{\left(A_{x_{1}} u\right.}\right)(\xi)-\widetilde{\left(A_{x_{2}} u\right.}\right)(\xi)=\left[A\left(x_{1}, \xi\right)-A\left(x_{2}, \xi\right)\right] \tilde{u}(\xi),
$$

which implies the inequality

$$
\begin{aligned}
\left\|\left(A_{x_{1}}-A_{x_{2}}\right) u\right\|_{l(x)}^{2} & =\int_{\mathbb{R}^{m}}\left|A\left(x_{1}, \xi\right)-A\left(x_{2}, \xi\right)\right|^{2}|\tilde{u}(\xi)|^{2}(1+|\xi|)^{2 l} d \xi \\
& \leq C\left|x_{1}-x_{2}\right|^{2} \int_{\mathbb{R}^{m}}|\tilde{u}(\xi)|^{2}(1+|\xi|)^{2(l(x)+\alpha(x))} d \xi=C\left|x_{1}-x_{2}\right|^{2}\|u\|_{l(x)-\alpha(x)}^{2} .
\end{aligned}
$$

Therefore,

$$
\left\|A_{x_{1}} u-A_{x_{2}} u\right\|_{l(x)} \leq C\left|x_{1}-x_{2}\right|\|u\|_{l(x)+\alpha(x)} .
$$

Further, by the properties of the norm, we have the estimate

$$
\left\|\left|A_{x_{1}} u\left\|_{l(x)}-\right\| A_{x_{2}} u\left\|_{l(x)} \mid \leq\right\| A_{x_{1}} u-A_{x_{2}} u \|_{l(x)},\right.\right.
$$

and hence, by inequality (6), the estimate

$$
\left\|\left|A_{x_{1}} u\left\|_{l(x)}-\right\| A_{x_{2}} u\left\|_{l(x)}|\leq C| x_{1}-x_{2} \mid\right\| u \|_{l(x)+\alpha(x)} .\right.\right.
$$

Now we take $l(x)=s\left(x_{2}\right)$ and $\alpha(x)=\alpha\left(x_{2}\right)$ and use the boundedness of the operator $A_{x_{2}}$ in the space $H^{s\left(x_{2}\right)}$ to obtain

$$
\left\|A_{x_{2}} u\right\|_{s\left(x_{2}\right)}-C\left|x_{1}-x_{2}\left\|\left|u\left\|_{s\left(x_{2}\right)+\alpha\left(x_{2}\right)} \leq\right\| A_{x_{1}} u\left\|_{s\left(x_{2}\right)} \leq\right\| A_{x_{2}} u\left\|_{s\left(x_{2}\right)}+C \mid x_{1}-x_{2}\right\|\|u\|_{s\left(x_{2}\right)+\alpha\left(x_{2}\right)} .\right.\right.\right.
$$


The last inequality implies that the operator $A_{x_{1}}$ is bounded as the operator $H^{s\left(x_{2}\right)} \rightarrow H^{s\left(x_{2}\right)-\alpha\left(x_{2}\right)}$ and the norm of this operator is close to the norm of the operator $A_{x_{2}}$ provided that the points $x_{1}$ and $x_{2}$ are close to each other. The proof of the theorem is complete.

Remark 3. Theorem 1 means that the property of local boundedness is preserved in a sufficiently small neighborhood of the point $x_{0}$.

\subsection{Examples}

1. The first example is from the theory of differential operators of fractional order. Consider a symbol of the form $[11,13]$

$$
A(x, \xi)=\left(1+\xi_{1}^{2}+\xi_{2}^{2}+\cdots+\xi_{m}^{2}\right)^{\alpha(x)} .
$$

This is the so-called fractional Laplacian of variable order. All conditions imposed above on the symbol are satisfied.

2. The second example is an integral operator of potential type (e.g., see [14] and references therein), or the hypersingular integral

$$
\left(I^{\alpha(x)} u\right)(x)=\int_{\mathbb{R}^{m}} \frac{c(x, y) u(y) d y}{|x-y|^{n-\alpha(x)}} .
$$

In the simplest case $(c(x, y) \equiv 1)$, the symbol of such an operator has the form (7), but apparently it can also be treated as the fractional Laplacian of variable order. In this connection, it should be noted that popular fractional objects can readily be described in the theory of pseudodifferential operators. Of course, pseudodifferential operators are rigidly related to the Fourier transform and special analysis is required in the cases where this technique cannot be applied.

\section{LOCAL PRINCIPLE AND FREDHOLMNESS}

Consider another representation of the operator $A$ with symbol $A(x, \xi)$ which is more convenient compared with the preceding one:

$$
(A u)(x)=\int_{\mathbb{R}^{m}} A(x, \xi) e^{i x \cdot \xi} \tilde{u}(\xi) d \xi .
$$

In this section, we subject the symbol $A(x, \xi)$ to stronger restrictions with respect to the variable $x$ to ensure the boundedness of the pseudodifferential operator $A$ in the space $H^{s_{M}}$.

\subsection{Boundedness Theorem}

Theorem 2. If an operator $A$ with symbol $A(x, \xi)$ is locally bounded, then it is bounded as the operator $A: H^{s_{M}} \rightarrow H^{(s-\alpha)_{m}}$.

Proof. By $C_{0}^{\infty}(\mathbb{R})$ we denote the set of infinitely differentiable compactly supported functions. Let $x_{0} \in \mathbb{R}^{m}$ be an arbitrary point, and let $\psi_{x_{0}}(x) \in C_{0}^{\infty}\left(\mathbb{R}^{m}\right)$ be a function defined in a neighborhood of the point $x_{0}$. Since the property of local boundedness of a pseudodifferential operator is preserved in a neighborhood $U\left(x_{0}\right)$ of the point $x_{0}$, it follows that this property holds for any point in this neighborhood; i.e., the operator

$$
\psi_{x_{0}}(x) A: H^{s(x)} \rightarrow H^{s(x)-\alpha(x)}, \quad x \in U\left(x_{0}\right),
$$

is bounded. Since the point $x_{0}$ is arbitrary, the latter property holds at any point $x \in \mathbb{R}^{m}$.

By $\dot{\mathbb{R}}^{m}$ we denote the one-point compactification of the space $\mathbb{R}^{m}$, and for each point $x_{0} \in \dot{\mathbb{R}}^{m}$ we construct a ball $U\left(x_{0}\right)$ centered at this point such that the property of local boundedness is satisfied at all of its points. Obviously, the union of all such balls forms a cover of the space $\dot{\mathbb{R}}^{m}$. 
By the compactness of $\dot{\mathbb{R}}^{m}$, we can choose a finite subcover $U\left(x_{k}\right), k=1, \ldots, n$, from this cover and assume that $\left\{\varphi_{k}(x)\right\}_{k=1}^{n}$ is the partition of unity corresponding to this cover. Then we have

$$
A=\sum_{k=1}^{n} \varphi_{k}(x) A
$$

and can apply the preceding result to each of the operators $\varphi_{k}(x) A$. The assertion of the theorem now follows from the inequalities

$$
\|A u\|_{(s-\alpha)_{m}} \leq\|A u\|_{s\left(x_{k}\right)-\alpha\left(x_{k}\right)} \leq C\|u\|_{s\left(x_{k}\right)} \leq C\|u\|_{s_{M}}, \quad k=1, \ldots, n .
$$

The proof of the theorem is complete.

Remark 4. Apparently, for Theorem 2 to hold, it suffices to require that the property of local boundedness be satisfied on a countable everywhere dense set of points (including the point at infinity).

\subsection{Completely Continuous (Compact) Operators in the Spaces $H^{s(x)}$}

Assume that a family of bounded linear operators $\left\{T_{x}: H^{s_{1}(x)} \rightarrow H^{s_{2}(x)}\right\}_{x \in \mathbb{R}^{m}}$ is given.

Lemma 4. If a continuous linear operator $T_{x_{0}}: H^{s_{1}\left(x_{0}\right)} \rightarrow H^{s_{2}\left(x_{0}\right)}$ is compact, then there exists a neighborhood $U_{x_{0}}$ of a point $x_{0}$ such that, for all $x_{1} \in U_{x_{0}}$, the operator $T_{x_{1}}: H^{s_{1}\left(x_{1}\right)} \rightarrow H^{s_{2}\left(x_{1}\right)}$ is compact as well.

Proof. To simplify the calculations, we assume that $s_{1}(x)=s_{2}(x)=s(x)$. By Lemma 2, the family of spaces $H^{s(x)}$ is locally continuous. We take an arbitrary bounded sequence $\left\{y_{n}\right\} \subset$ $H^{s\left(x_{1}\right)},\left\|y_{n}\right\|_{s\left(x_{1}\right)} \leq C$, and fix a sufficiently small number $\varepsilon>0$. For each $y_{n} \in H^{s\left(x_{1}\right)}$, we choose a function $v_{n} \in S\left(\mathbb{R}^{m}\right)$ so that the inequality $\left\|y_{n}-v_{n}\right\|_{s\left(x_{1}\right)}<\varepsilon$ is satisfied. Since $v_{n}=v_{n}-y_{n}+y_{n}$, we obtain

$$
\left\|v_{n}\right\|_{s\left(x_{1}\right)} \leq\left\|v_{n}-y_{n}\right\|_{s\left(x_{1}\right)}+\left\|y_{n}\right\|_{s\left(x_{1}\right)}<C+\varepsilon,
$$

and hence the sequence $\left\{v_{n}\right\}$ is bounded in the space $H^{s\left(x_{1}\right)}$. But then, by Lemma 1 , this sequence is also bounded in the space $H^{s\left(x_{0}\right)}$ if the points $x_{0}$ and $x_{1}$ are sufficiently close to each other, $\left\|v_{n}\right\|_{s\left(x_{0}\right)} \leq 2 C$.

Since $T$ is a bounded linear operator in the space $H^{s\left(x_{0}\right)}$, the sequence $z_{n}=T v_{n}$ is also bounded in this space. By the compactness of the operator $T$ in the space $H^{s\left(x_{0}\right)}$, the sequence $\left\{z_{n}\right\}$ contains a subsequence $\left\{z_{n_{k}}\right\}$ converging in $H^{s\left(x_{0}\right)}$, so that

$$
\left\|z_{0}-z_{n_{k}}\right\|_{s\left(x_{0}\right)} \rightarrow 0 \quad \text { as } \quad k \rightarrow \infty, \quad z_{0} \in H^{s\left(x_{0}\right)} .
$$

By the completeness of the space $H^{s\left(x_{0}\right)}$, the sequence $\left\{z_{n_{k}}\right\}$ is fundamental. By definition, this means that for any $\varepsilon>0$ there exists an $N$ such that the inequality $\left\|z_{k_{N}}-z_{k_{N+p}}\right\|_{s\left(x_{0}\right)}<\varepsilon$ holds for all $n \geq N$ and $p>0$.

Further, since the space $S\left(\mathbb{R}^{m}\right)$ is everywhere dense in any space $H^{s(x)}$, we choose elements $v_{k_{N}}$, $v_{k_{N+p}} \in S\left(\mathbb{R}^{m}\right)$ so to satisfy the inequalities

$$
\left\|z_{k_{N}}-v_{k_{N}}\right\|_{s\left(x_{0}\right)}<\varepsilon / 2, \quad\left\|z_{k_{N+p}}-v_{k_{N+p}}\right\|_{s\left(x_{0}\right)}<\varepsilon / 2 .
$$

In other words, the sequences $\left\{z_{n_{k}}\right\} \subset H^{s\left(x_{0}\right)}$ and $\left\{v_{n_{k}}\right\} \subset S\left(\mathbb{R}^{m}\right)$ are cofinal with respect to the norm of the space $H^{s\left(x_{0}\right)}$. By Lemma 1 ,

$$
\left\|\left|v_{k_{N}}-v_{k_{N+p}}\left\|_{s\left(x_{1}\right)}-\right\| v_{k_{N}}-v_{k_{N+p}}\left\|_{s\left(x_{0}\right)}|<| x_{1}-x_{0}\left|\left\|z_{k_{N}}-z_{k_{N+p}}\right\|_{s_{M}} \leq\|T\| C_{1}\right| x_{1}-x_{0} \mid,\right.\right.\right.
$$

so that if, as a neighborhood of the point $x_{0}$, we take a ball $U\left(x_{0}, \varepsilon_{1}\right)$ centered at $x_{0}$ of radius $\varepsilon_{1}<\varepsilon /\left(C_{1}\|T\|\right)$, then the inequality

$$
\left\|v_{k_{N}}-v_{k_{N+p}}\right\|_{s\left(x_{1}\right)}<\varepsilon
$$

DIFFERENTIAL EQUATIONS $\quad$ Vol. $54 \quad$ No. $9 \quad 2018$ 
holds any $x_{1} \in U\left(x_{0}, \varepsilon_{1}\right)$, which implies that the subsequence $\left\{v_{n_{k}}\right\}$ is fundamental (and converges by the completeness) with respect to the norm of the space $H^{s\left(x_{1}\right)}$. The proof of the lemma is complete.

Let $\psi_{x_{0}}(x) \in C_{0}^{\infty}\left(\mathbb{R}^{m}\right)$ be a function equal to 1 in a neighborhood of the point $x_{0}$.

Corollary 1. The operator with symbol $\psi_{x_{0}}(x) A(x, \xi)-A\left(x_{0}, \xi\right)$ is completely continuous in a neighborhood of the point $x_{0}$ as an operator $H^{s(x)} \rightarrow H^{s(x)-\alpha(x)}$.

Proof. Consider a family of pseudodifferential operators with symbol $\psi_{x_{0}}(x) A(x, \xi)-A\left(x_{0}, \xi\right)$, where the point $x_{0} \in \mathbb{R}^{m}$ is fixed. By inequality (4), this operator $H^{s(x)} \rightarrow H^{s(x)-\alpha(x)}$ is bounded in a neighborhood of the point $x_{0}$ and is zero at the point $x_{0}$, and hence it is completely continuous. Then, by Theorem 4, there exists a neighborhood of the point $x_{0}$ such that, for all points $x$ in this neighborhood, the operator $H^{s(x)} \rightarrow H^{s(x)-\alpha(x)}$ is completely continuous. The proof of the corollary is complete.

\subsection{Local Representatives and Conditions for Being Fredholm}

Definition 8. An operator $A_{x_{0}}$ with symbol $A\left(x_{0}, \xi\right)\left(x_{0} \in \mathbb{R}^{m}\right.$ is fixed) is called a local representative of the operator $A$ at the point $x_{0}$.

(This term and the whole ideology of transition from local to global (with insignificant losses) were proposed by Simonenko in the mid-1960s; see [21]; here we slightly modify his argument and change its order.)

Theorem 3. If the local representatives $A_{x_{0}}: H^{s\left(x_{0}\right)} \rightarrow H^{s\left(x_{0}\right)-\alpha\left(x_{0}\right)}$ of the operator $A$ are invertible at each point of the space $\mathbb{R}^{m}$ including the point at infinity, then $A: H^{s_{M}} \rightarrow H^{(s-\alpha)_{m}}$ is a Fredholm operator.

Proof. To prove the Fredholm property of being for the operator under study, it suffices to construct a so-called regularizer (sometimes called an almost inverse operator), i.e., an operator $C$ such that

$$
A C=I+T_{1}, \quad C A=I+T_{2},
$$

where $I$ is the identity operator and $T_{1}$ and $T_{2}$ are compact operators.

By the properties of the symbol, the function $A(x, \xi)$ is continuous in $x$ on the compactification $\dot{\mathbb{R}}^{m}$ of the space $\mathbb{R}^{m}$, i.e., there exists a limit $\lim _{|x| \rightarrow+\infty} \equiv A(\infty, \xi)$. We distinguish a neighborhood $U(\infty) \subset \mathbb{R}^{m}$ of the point at infinity(such a neighborhood is understood as the exterior of a ball centered at the origin of sufficiently large radius) such that inequality $\left(4^{\prime}\right)$ holds for all $x \in U(\infty)$. The set $\mathbb{R}^{m} \backslash U(\infty)$ is compact. For each point $x \in \mathbb{R}^{m} \backslash U(\infty)$, we choose an open ball $U(x)$ centered at that point such that inequality (4) holds for all points of the ball. Obviously, the family $U(x)$, $x \in \mathbb{R}^{m} \backslash U(\infty)$, forms an open cover of the set $\mathbb{R}^{m} \backslash U(\infty)$. By the compactness of $\mathbb{R}^{m} \backslash U(\infty)$, this cover contains a finite subcover, which we denote by $\bigcup_{k=1}^{n-1} U\left(x_{k}\right)$. To this cover, we add a neighborhood $U(\infty)$. For the finite cover $\bigcup_{k=1}^{n-1} U\left(x_{k}\right) \bigcup U(\infty)$, there exists a partition of unity subordinate to this cover, which we denote by $\left\{\varphi_{k}(x)\right\}_{k=1}^{n}$. For each function $\varphi_{k}(x)$, we construct a compactly supported function $\psi_{k}(x) \in C_{0}^{\infty}\left(\mathbb{R}^{m}\right)$ such that $\psi_{k}(x) \equiv 1$ on the support of $\varphi_{k}(x)$ and the supports of $\varphi_{k}(x)$ and $1-\psi_{k}(x)$ are disjoint. We represent the operator $A$ as

$$
A=\sum_{k=1}^{n} \varphi_{k}(x) A=\sum_{k=1}^{n} \varphi_{k}(x) A \psi_{k}(x)+T,
$$

where the operator $T$ has the form

$$
T=\sum_{k=1}^{n} \varphi_{k}(x) A\left(1-\psi_{k}(x)\right) .
$$

We write $A_{k}=\varphi_{k} A \psi_{k}$. Since the variable $x$ of the symbol $A(x, \xi)$ varies in the neighborhood $U\left(x_{k}\right)$, it follows from Theorem 1 that the operator $A_{k}: H^{s(x)}\left(U_{k}\right) \rightarrow H^{s(x)-\alpha(x)}\left(U_{k}\right)$ is 
bounded for any $x \in U_{k}$. In other words, we have the estimate

$$
\left\|A_{k} u\right\|_{s(x)} \leq C_{k}\|u\|_{s(x)-\alpha(x)}, \quad x \in U_{k} .
$$

Further, we write

$$
A_{k}=\varphi_{k}\left(A-A_{x_{k}}\right) \psi_{k}+\varphi_{k} A_{x_{k}} \psi_{k},
$$

so that, by Corollary 1 , the first term is a completely continuous operator, and the operator $A_{x_{k}}$ is invertible by the condition of the theorem. Each of the operators $\varphi_{j}(x) A\left(1-\psi_{k}(x)\right)$ is completely continuous (the kernel of the operator has no singularity by the condition $|x-y| \geq c$ ), and hence each of such operators is a Hilbert-Schmidt operator in any space $H^{s}$ [4, p. 171]; therefore, it is completely continuous. Thus, the operator $A$ can be written as

$$
A=\sum_{k=1}^{n} \varphi_{k}(x) A_{x_{k}} \psi_{k}(x)+T \text {. }
$$

Now we introduce an operator $C$ as a pseudodifferential operator with symbol $A^{-1}(x, \xi)$ and show that this operator is a regularizer for the operator $A$. We multiply (9) by the operator $C$ on the left and obtain

$$
C A=C \sum_{k=1}^{n} \varphi_{k}(x) A_{x_{k}} \psi_{k}(x)+T_{1},
$$

where $T_{1}=C T$, and further,

$$
C A=\sum_{k=1}^{n} \psi_{k}(x) C \varphi_{k}(x) A_{x_{k}} \psi_{k}(x)+T_{2},
$$

because the operator $C$ and its symbol $C(x, \xi)=A^{-1}(x, \xi)$ have properties similar to the properties of the operator $A$ and the symbol $A(x, \xi)$. Then we introduce the operator $C_{k}=\psi_{k}(x) C \varphi_{k}(x)$ and, by analogy with (8), obtain $C_{k}=\psi_{k}\left(C-C_{x_{k}}\right) \varphi_{k}+\psi_{k} C_{x_{k}} \varphi_{k}$.

Thus,

$$
C A=\sum_{k=1}^{n} \psi_{k}(x) C_{x_{k}} \varphi_{k}(x) A_{x_{k}} \psi_{k}(x)+T_{3},
$$

Further, we apply the property of "almost commutativity" between the operator of multiplication by the function $\varphi_{k}(x)$ and the operator $A_{x_{k}}$, which means that the operator $\varphi_{k}(x) A_{x_{k}}-A_{x_{k}} \varphi_{k}(x)$ is completely continuous in any space $H^{s}[4$, p. 49]. With this taken into account, we obtain

$$
C A=\sum_{k=1}^{n} \psi_{k}(x) C_{x_{k}} A_{x_{k}} \varphi_{k}(x) \psi_{k}(x)+T_{4}=\sum_{k=1}^{n} \varphi_{k}(x)+T_{4}=I+T_{4},
$$

because $\varphi_{k}(x) \psi_{k}(x)=\varphi_{k}(x)$ and $\sum_{k=1}^{n} \varphi_{k}(x)=1$.

One can verify in a similar way that the product $A C$ is the sum of the identity operator and a completely continuous operator. The proof of the theorem is complete.

Remark 5. Under the assumptions of Theorem 3, it is apparently also sufficient to require the invertibility of local representatives on an everywhere dense set of points.

\section{THE CASE OF HALF-SPACE}

Definition 9. Let a function $A(x, \xi)$ locally integrable with respect to $\xi$ be given on $\mathbb{R}_{+}^{m} \times \mathbb{R}^{m}$. A pseudodifferential operator $A$ in the half-space with symbol $A(x, \xi)$ is an operator of the form

$$
(A u)(x)=\int_{\mathbb{R}_{+}^{m}}\left(\int_{\mathbb{R}^{m}} A(x, \xi) e^{i(x-y) \xi} u(y) d \xi\right) d y, \quad x \in \mathbb{R}_{+}^{m} .
$$


The function $A(x, \xi)$ is called the symbol of the operator $A$.

Such an operator is associated with the following equation in the half-space:

$$
(A u)(x)=v(x), \quad x \in \mathbb{R}_{+}^{m} .
$$

Following [4, p. 43], we introduce the space $H^{s(x)}\left(\mathbb{R}_{+}^{m}\right)$ of functions in $H^{s(x)}\left(\mathbb{R}^{m}\right)$ supported in the closed half-space $\overline{\mathbb{R}_{+}^{m}}$, where we seek the solution, and the space $H_{0}^{s(x)}\left(\mathbb{R}_{+}^{m}\right)$ of (generalized) functions in the space $S^{\prime}\left(\mathbb{R}^{m}\right)$ supported in the half-space $\overline{\mathbb{R}_{+}^{m}}$ and admitting continuation to the entire $H^{s(x)}\left(\mathbb{R}^{m}\right)$, with finite norm

$$
\|v\|_{s(x)}^{+}=\inf \|\ell v\|_{s(x)},
$$

where the infimum is taken over all possible continuations $\ell$.

To study the Fredholm property of Eq. (11), we apply the general methodology of local principle $[21$, p. 13] just as it is used to study equations on manifolds. We distinguish two types of local representatives.

1. For an interior point $x_{0} \in \mathbb{R}_{+}^{m}$, this is the operator considered here:

$$
\left(A_{x_{0}} u\right)(x)=\iint_{\mathbb{R}^{m}} \int_{\mathbb{R}^{m}} A\left(x_{0}, \xi\right) e^{i(x-y) \xi} u(y) d y d \xi, \quad x \in \mathbb{R}^{m} .
$$

2. For a boundary point $x_{0}^{\prime}=\left(x_{0}^{\prime}, 0\right) \in \mathbb{R}^{m-1}$ (for $x_{0} \in \mathbb{R}^{m}$, we use the notation $x_{0}=\left(x_{0}^{\prime}, x_{m}^{(0)}\right)$, where $\left.x_{m}^{(0)} \in \mathbb{R}\right)$, this is the operator

$$
\left(A_{x_{0}^{\prime}} u\right)(x) \mapsto \int_{\mathbb{R}_{+}^{m}}\left(\int_{\mathbb{R}^{m}} A\left(x_{0}^{\prime}, \xi\right) e^{i(x-y) \xi} u(y) d \xi\right) d y, \quad x \in \mathbb{R}_{+}^{m} .
$$

Thus, we have two families of operators.

Theorem 4. If all operators of the family $A_{x_{0}^{\prime}}: H^{s\left(x^{\prime}\right)}\left(\mathbb{R}_{+}^{m}\right) \rightarrow H^{s\left(x^{\prime}\right)-\alpha\left(x^{\prime}\right)}\left(\mathbb{R}_{+}^{m}\right)$ are bounded, then the operator $(10)$ is bounded as an operator $H^{s_{M}}\left(\mathbb{R}_{+}^{m}\right) \rightarrow H^{(s-\alpha)_{m}}\left(\mathbb{R}_{+}^{m}\right)$. If all operators of the families are invertible, then the operator (10) is Fredholm as an operator $H^{s_{M}}\left(\mathbb{R}_{+}^{m}\right) \rightarrow H^{(s-\alpha)_{m}}\left(\mathbb{R}_{+}^{m}\right)$.

The scheme of proof is similar to that described above with the only difference that it is now necessary to consider each of the two different families of local operators separately.

\subsection{Variable Factorization Index}

The key role in studying the solvability of Eq. (11) in the classical Sobolev-Slobodetskii spaces is played by the factorization index of the elliptic symbol. We give the corresponding definition for our case and show how it can be used to study the invertibility of the operator (12) with "frozen" pole $x^{\prime} \in \mathbb{R}^{m-1}$.

Definition 10. The factorization of a symbol $A\left(x^{\prime}, \xi\right)$ with respect to the variable $\xi_{m}$ is its representation in the form

$$
A\left(x^{\prime}, \xi^{\prime}, \xi_{m}\right)=A_{+}\left(x^{\prime}, \xi^{\prime}, \xi_{m}\right) A_{-}\left(x^{\prime}, \xi^{\prime}, \xi_{m}\right),
$$

where the factors $A_{+}\left(A_{-}\right)$can analytically be continued in $\xi_{m}$ into the upper (lower) complex half-plane of the variable $\xi_{m} \pm i \tau, \tau>0$, for almost all $\xi^{\prime} \in \mathbb{R}^{m-1}$ and satisfy the estimates

$$
\begin{aligned}
& \left|A_{+}\left(x^{\prime}, \xi^{\prime}, \xi_{m}\right)\right| \leq c_{1}\left(1+\left|\xi^{\prime}\right|+\left|\xi_{m}\right|+|\tau|\right)^{\varkappa\left(x^{\prime}\right)}, \\
& \left|A_{-}\left(x^{\prime}, \xi^{\prime}, \xi_{m}\right)\right| \leq c_{1}\left(1+\left|\xi^{\prime}\right|+\left|\xi_{m}\right|+|\tau|\right)^{\alpha\left(x^{\prime}\right)-\varkappa\left(x^{\prime}\right)}
\end{aligned}
$$

for all $\tau \in \mathbb{R}$. The function $\varkappa\left(x^{\prime}\right), x^{\prime} \in \mathbb{R}^{m-1}$, is called a variable factorization index. 
Since the value of the factorization index determines the solvability pattern of the simplest equation (11) (for a constant index, this is well known $[4$, p. 71; 18]), we consider one of the most interesting cases below. Note that such a factorization can constructively be obtained by using Cauchy type integrals (see $[4,11,13]$ ).

Throughout the following, we assume that the function $\varkappa\left(x^{\prime}\right)$ has the same properties as the function $s\left(x^{\prime}\right)$.

\subsection{Local Invertibility and the Conditions for the Fredholm Property}

The problem of the applicability conditions naturally arises for Theorem 4. In other words, it is required to understand under what conditions the local representatives of the "boundary" operator are invertible.

Theorem 5. Let

$$
\left|\varkappa\left(x^{\prime}\right)-s\left(x^{\prime}\right)\right|<1 / 2 \quad \text { for any } \quad x^{\prime} \in \mathbb{R}^{m-1} .
$$

Then the operator $(12)$ is invertible as an operator $H^{s\left(x^{\prime}\right)}\left(\mathbb{R}_{+}^{m}\right) \rightarrow H^{s\left(x^{\prime}\right)-\alpha\left(x^{\prime}\right)}\left(\mathbb{R}_{+}^{m}\right)$ for each fixed $x^{\prime}$.

The proof of Theorem 5 completely reproduces the corresponding argument for the constant factorization index in the monograph [4, p. 74].

A combination of Theorems 4 and 5 implies the following result about the Fredholm property of a pseudodifferential operator (equation) in a half-space.

Theorem 6. Let $A(x, \xi) \in E_{\alpha(x)}$. If inequality (13) is satisfied, then the operator (10) is Fredholm as an operator $H^{s_{M}}\left(\mathbb{R}_{+}^{m}\right) \rightarrow H^{(s-\alpha)_{m}}\left(\mathbb{R}_{+}^{m}\right)$.

\subsection{Boundary Operators and Boundary Value Problems}

If condition (13) is violated, then the assertion of Theorem 5 is, generally speaking, not true. Several scenarios of solvability can be found in $[4$, pp. $75-78 ; 13]$. Here we consider only one possible version.

Let $\varkappa\left(x^{\prime}\right)-s\left(x^{\prime}\right)=n+\delta,|\delta|<1 / 2, n \in \mathbb{N}$, for all $x^{\prime} \in \mathbb{R}^{m-1}$. Now even for a fixed pole $x^{\prime}$ the operator (12) is not invertible, but the structure of the general solution of the corresponding equation is known [4, p. 76]. Let us briefly describe this structure to explain the appearance of boundary operators.

Thus, we consider an equation with the operator (12) and right-hand side $f \in H_{0}^{s\left(x_{0}^{\prime}\right)-\alpha\left(x_{0}^{\prime}\right)}\left(\mathbb{R}_{+}^{m}\right)$. The general solution of the equation

$$
\left(A_{x_{0}^{\prime}} u\right)(x)=f(x), \quad x \in \mathbb{R}_{+}^{m}
$$

with the operator (12) (recall that $x_{0}^{\prime} \in \mathbb{R}^{m-1}$ is fixed) in Fourier transforms has the form [4, p. 75]

$$
\tilde{u}(\xi)=A_{+}^{-1}\left(x_{0}^{\prime}, \xi\right) P_{n}(\xi) \Pi_{+} P_{n}^{-1}(\xi) A_{-}^{-1}\left(x_{0}^{\prime}, \xi\right) \widetilde{\ell f}(\xi)+A_{+}^{-1}\left(x_{0}^{\prime}, \xi\right) \sum_{k=1}^{n} \tilde{c}_{k}\left(x_{0}^{\prime}, \xi^{\prime}\right) \xi_{m}^{k-1},
$$

where $\Pi_{+}$is the Cauchy type integral

$$
\left(\Pi_{+} \tilde{u}\right)(\xi)=\frac{i}{2 \pi} \lim _{\tau \rightarrow 0+} \int_{-\infty}^{+\infty} \frac{\tilde{u}\left(\xi^{\prime}, \eta_{m}\right)}{\xi_{m}-\eta_{m}+i \tau} d \eta_{m},
$$

$P_{n}(\xi) \in E_{n}$ is an arbitrary polynomial (see Definition 6), and the $c_{k}\left(x_{0}^{\prime}, \xi^{\prime}\right)$ are arbitrary functions in the space $H^{s_{k}\left(x_{0}^{\prime}\right)}\left(\mathbb{R}^{m-1}\right), s_{k}\left(x_{0}^{\prime}\right)=s\left(x_{0}^{\prime}\right)-\varkappa\left(x_{0}^{\prime}\right)+k-1 / 2, k=1, \ldots, m$. In particular, it follows that in this situation the second type of local representative given by formula (12) is chosen poorly; i.e., it is never invertible. Thus, we need a new type of local representative to be able to apply 
Theorem 4. Moreover, we also need new local Sobolev-Slobodetskii spaces where the new local representative will act.

To determine the functions $c_{k}$ uniquely, we introduce $m$ bounded pseudodifferential operators $B_{j}: H^{s\left(x_{0}^{\prime}\right)}\left(\mathbb{R}_{+}^{m}\right) \rightarrow H^{s\left(x_{0}^{\prime}\right)-\alpha_{j}\left(x_{0}^{\prime}\right)}\left(\mathbb{R}_{+}^{m}\right)$ with symbols $B_{j}\left(x_{0}, \xi\right), j=1, \ldots, n$ and let $\gamma$ denote the operator of restriction to the hyperplane $x_{m}=0$ so that the operators $\gamma B_{j}: H^{s\left(x_{0}^{\prime}\right)}\left(\mathbb{R}_{+}^{m}\right) \rightarrow$ $H^{s\left(x_{0}^{\prime}\right)-\alpha_{j}\left(x_{0}^{\prime}\right)-1 / 2}\left(\mathbb{R}^{m-1}\right)$ are bounded by the condition $s\left(x_{0}^{\prime}\right)-\alpha_{j}\left(x_{0}^{\prime}\right)-1 / 2>0, x_{0}^{\prime} \in \mathbb{R}^{m-1}[13]$. We define a new local Sobolev-Slobodetskii space as the direct sum

$$
H^{s_{\alpha}\left(x_{0}^{\prime}\right)} \equiv H^{s\left(x_{0}^{\prime}\right)}\left(\mathbb{R}_{+}^{m}\right) \bigoplus \sum_{j=1}^{m} H^{s\left(x_{0}^{\prime}\right)-\alpha_{j}\left(x_{0}^{\prime}\right)-1 / 2}\left(\mathbb{R}^{m-1}\right) .
$$

For a point $x_{0}^{\prime} \in \mathbb{R}^{m-1}$, we introduce the local operator $H^{s\left(x_{0}^{\prime}\right)}\left(\mathbb{R}_{+}^{m}\right) \rightarrow H^{s_{\alpha}\left(x_{0}^{\prime}\right)}$ by the relation

$$
B_{x_{0}^{\prime}} u=\left(A_{x_{0}^{\prime}} u, \gamma B_{1} u, \gamma B_{2} u, \ldots, \gamma B_{m} u\right) .
$$

Thus, the operator $B_{x_{0}^{\prime}}$ is obtained by "gluing" $m$ additional operators together with the family of additional spaces to the operator $A_{x_{0}^{\prime}}$ at each point $x_{0}^{\prime} \in \mathbb{R}^{m-1}$.

Under the above assumptions on the symbols, as above, we conclude that all operators are locally bounded and the local boundedness implies the boundedness of the operator (14).

The invertibility of the local operator (14) can be investigated by using the Fourier transform, which permits reducing the problem of identifying arbitrary functions $c_{k}$ to the problem of unique solvability of an $m \times m$ system of linear algebraic equations. The nonvanishing of the determinant of this system is a necessary and sufficient condition for the invertibility of the local operator $B_{x_{0}^{\prime}}$.

Remark 6. There is one case left, where $\varkappa\left(x^{\prime}\right)-s\left(x^{\prime}\right)=-n+\delta,|\delta|<1 / 2, n \in \mathbb{N}$, for all $x^{\prime} \in \mathbb{R}^{m-1}$. It can mainly be considered as above, only the operator $A_{x^{\prime}}$ has an extended domain of definition to which additional local Sobolev-Slobodetskii spaces with additional unknowns in these spaces are glued.

\section{CONCLUSION}

Apparently, most of the results obtained by using the local principle can be transferred to operators and equations of variable order. Some results can be found in $[12,13]$ for the cases of the space $\mathbb{R}^{m}$ and the half-space $\mathbb{R}_{+}^{m}$, but a different class of symbols is considered there. Moreover, it is of course of interest to study elliptic pseudodifferential equations and related boundary value problems in spaces of variable order for domains (manifolds) with singularities on the boundary [19, 20, $22-24]$.

\section{ACKNOWLEDGMENTS}

This work was supported by the Ministry of Education and Science of the Russian Federation (project no. 1.7311.2017/8.9).

\section{REFERENCES}

1. Taylor, M., Pseudodifferential Operators, Princeton: Princeton Univ., 1983.

2. Rempel, S. and Schulze, B., Index Theory of Elliptic Boundary Problems, Berlin: Akademie-Verlag, 1984.

3. Treves, F., Introduction to Pseudodifferential and Fourier Integral Operators, New York: Plenum, 1980.

4. Eskin, G.I., Kraevye zadachi dlya ellipticheskikh psevdodifferentsial'nykh uravnenii (Boundary Value Problems for Elliptic Pseudodifferential Equations), Moscow: Mosk. Gos. Univ., 1973.

5. Nazarov, S.A. and Plamenskii, B.A., Ellipticheskie zadachi v oblastyakh s kusochno gladkoi granitsei (Elliptic Problems in Domains with Piecewise Smooth Boundary), Moscow: Nauka, 1991.

6. Schulze, B.-W., Sternin, B., and Shatalov, V. Differential Equations on Singular Manifolds: Semiclassical Theory and Operator Algebras, Berlin-New York: Wiley-VCH, 1998. 
7. Nazaikinskii, V., Schulze, B.-W., and Sternin, B., The Localization Problem in Index Theory of Elliptic Operators, Basel: Springer, 2014.

8. Vasilyev, V.B., Multiplikatory integralov Fur'e, psevdodifferential'nye uravneniya, volnovaya faktorizatsiya, kraevye zadachi (Fourier Integral Multipliers, Pseudodifferential Equations, Wave Factorization, and Boundary Value Problems), Moscow: KomKniga, 2010.

9. Vladimirov, V.S., Metody teorii funktsii mnogikh kompleksnykh peremennykh (Methods of the Theory of Functions of Several Complex Variables), Moscow: Nauka, 1964.

10. Vasilyev, V.B., Regularization of multidimensional singular integral equations in nonsmooth domains, Trudy Moskov. Mat. Obs., 1998, vol. 59, pp. 73-105.

11. Vishik, M.I. and Eskin, G.I., Elliptic equations in convolution in a bounded domain and their applications, Russ. Math. Surveys, 1967, vol. 22, no. 1, pp. 13-75.

12. Volevich, L.R. and Kagan, V.M., Pseudodifferential hypoelliptic operators in the theory of function spaces, Trudy Moskov. Mat. Obshch., 1969, vol. 20, pp. 241-275.

13. Novozhilova, L.S., Sobolev-Slobodetskii spaces of variable order in an unbounded domain, in Theory of Functions, Functional Analysis, and Their Applications, Kharkov: Kharkov Gos. Univ., 1974, no. 20, pp. $122-133$.

14. Vakulov, B.G. and Drobotov, Yu.E., On weighted generalized variable Hölder spaces, Sib. Elektron. Mat. Izv., 2017, vol. 14, pp. 647-656.

15. Kryakvin, V.D., Boundedness of pseudodifferential operators in Hölder-Zygmund spaces of variable order, Sib. Math. J., 2014, vol. 55, no. 6, pp. 1073-1083.

16. Sharapudinov, I.I., Sobolev space with variable index and approximation by algebraic-trigonometric polynomials, Vestnik. Dagestan. Nauch. Tsentra, 2014, no. 53, pp. 5-21.

17. Shubin, M.A., Psevdodifferentsial'nye operatory i spectral'naya teoriya (Pseudodifferential Operators and Spectral Theory), Moscow: Nauka, 1978.

18. Vishik, M.I. and Eskin, G.I., Equations in convolutions in a bounded region, Russ. Math. Surveys, 1965, vol. 20, no. 3, pp. 85-151.

19. Vasilyev, V.B., Pseudodifferential equations on manifolds with complex singularities on the boundary, Sib. Zh. Chistoi i Prikl. Mat., 2016, no. 3, pp. 3-14.

20. Vasilyev, V.B., Model elliptic boundary-value problems for pseudodifferential equations in canonical nonsmooth domains, Trudy Sem. Petrovsk., 2016, vol. 31, pp. 22-37.

21. Simonenko, I.B., Lokal'nyi metod v teorii invariantnykh otnositel'no sdviga operatorov $i$ ikh ogibayushchikh (Local Method in the Theory of Translation-Invariant Operators and Their Envelopes), Rostovon-Don: TsVVR, 2007.

22. Vasilyev, V.B., Asymptotical analysis of singularities for pseudo differential equations in canonical nonsmooth domains, in Integral Methods in Science and Engineering. Computational and Analytic Aspects, Ed. by Constanda, C. and Harris, P.J., Boston, 2011, pp. 379-390.

23. Vasilyev, V.B., Potentials for elliptic boundary value problems in cones, Sib. Elektron. Mat. Izv., 2016, vol. 13, pp. 1129-1149.

24. Vasilyev, V.B., Pseudodifferential equations in cones with conjugation points on the boundary, Differ. Equations, 2015, vol. 51, no. 9, pp. 1113-1125. 